\title{
Social epigenetics: a science of social science?
}

Emma Chung ${ }^{\mathrm{a}}$, John Cromby ${ }^{\mathrm{b}}$, Dimitris Papadopoulos ${ }^{\mathrm{c}}$, and Cristina Tufarelli ${ }^{\mathrm{d}}$

${ }^{a}$ College of Medicine, Biological Sciences and Psychology, University of Leicester, Leicester Royal Infirmary, LE1 5WW, UK (emlc1@le.ac.uk)

b School of Management, University of Leicester, LE1 7RH, UK (john.cromby@le.ac.uk)

c School of Sociology and Social Policy, University of Nottingham, NG7 2RD, UK (dimitris.papadopoulos@nottingham.ac.uk)

d School of Medicine, University of Nottingham, Royal Derby Hospital, Derby DE22 3DT, UK (cristina.tufarelli@nottingham.ac.uk)

Sociological Review

2016

DOI: 10.1111/1467-954X.12019

\section{$\underline{\text { Abstract }}$}

Epigenetics has considerable potential to transform social science by embedding mutually regulative reciprocal connections between biological and social processes within the human activities it studies. This paper highlights common epigenetic methods and outlines practical considerations in the design of 'social epigenetics' research addressing the identification of biomolecular pathways, statistical inference of causality, conceptualisation of the environment as a biochemical event, heritability of epigenetic alterations and intergenerational accountability, and concept of time implied by attempts to capture complex, non-linear gene-environment interactions. Finally, we reflect on the social epigenome as a conceptual space and try to identify barriers to translation, and practical and ethical issues raised by epigenetics research. In order for social epigenetics and social science to contribute to the emergence of this putative 'science of social science' and to capture meaningful human experience they will both need to change significantly.

\section{$\underline{\text { Keywords }}$}

Social Epigenetics; Biomolecular Pathways; Temporality; Environment as Biochemical Event; Experience; Intergenerational Accountability; Reversibility 


\section{The emergence of epigenetics}

Despite initial expectations of DNA as containing all of the information required to characterise a specific individual (or phenotype), researchers quickly recognised that the information contained in our 23,000 genes provided a very limited explanation of individual difference and ontogenetic development. Over the past decade, a supplementary set of theories and experiments exploring the unpredictable dynamics of gene expression have begun to take centre stage in genetics research. These theories revisit a latent branch of biology known as 'epigenetics', first defined by Waddington in 1942 as the study of 'the causal interactions between genes and their products, which bring the phenotype into being' (Waddington, 2012).

The 'epigenome' describes the overall state of a cell in flux, each point in time yielding multiple cascading possibilities for divergence of individual phenotypes. The inert genome is thereby supplemented by a softer, more adaptable, epigenome, incorporating mechanisms capable of responding to the environment, sensing 'time' and retaining 'memories' that regulate subsequent development. Within myriad microscopic epigenomes, the effects of the wider social and physical environment are translated via biochemical interactions to become an integral part of a fluctuating landscape of gene expression. But how can these be studied, in particular, the interactions between the social world and the epigenome? Which research methodologies are necessary for exploring this supposed 'science of social science'? This paper briefly introduces some basic concepts in epigenetics and explores the conceptual space offered by the epigenome. We examine research foci and existing methodologies used to underpin epigenetics research, and discuss the challenges of translating these to a social sciences arena. Finally, we discuss opportunities and obstacles to the flow of knowledge within this uniquely multi-disciplinary, and rapidly evolving, area of research.

\section{Visualising the epigenome}

In thinking about our genetic identity via epigenetics we are invited to project ourselves down to the bio-molecular level of DNA. Visual metaphors include Waddington's original illustrations, in which the ridges and furrows of the epigenetic landscape are anchored to genes via interconnected ropes (Waddington, 2012), or depiction of fluctuations in gene expression as an array of flashing lights or switches, however, a shared vision of the active epigenome has yet to be established. In some descriptions, DNA is described as computer hardware, which is supplemented by epigenetic 'software' (Hoffmann \& Spengler, 2012). The dynamic, functional character of the epigenome has also been likened to interpretation of a play, cooking recipe, or musical score (Jablonka \& Lamb, 2005, p. 118), but none of these metaphors seem to satisfactorily conjure the agency of environment. Although the epigenetics 'revolution' has been turning for over a decade, epigenetics has yet to gain significant traction within broader academic research as well as popular imagination. This is no doubt in part because the purported distinctiveness and revolutionary potentials of epigenetics are questioned even by some of its most eminent protagonists (Pickersgill, this volume). Such questioning clearly illustrates how concepts and definitions underlying what is meant by epigenetics are still in the process of standardisation amongst biomedical scientists, and are tentatively being accepted and absorbed by researchers outside of the field. This lack of widely accepted visual representations of the 
epigenome is revealing that a definite orientation of the field is yet to be found and is symptomatic of the absence of broadly accepted principles.

All of the cells in our body share identical DNA, but the appearance and function of cells radically diverge during development, governed by a cascade of interactions that lead some parts of the genome to become more influential than others. The choice of which regions of our DNA become more or less influential is regulated by epigenetic alterations, which accumulate during development and are perpetuated by future cells of the organ without any underlying change in the DNA sequence. Thus, as the fertilised egg evolves from embryo to foetus, neonate, infant, adolescent, and adult, a single genome can be thought of as branching into numerous distinct epigenomes.

The 'epigenome' can therefore be regarded as a conceptual space encompassing the functional interpretation of genetic material within and between cells. The epigenome is intrinsically less rigid than the genome, reacting to environmental cues, and allowing specific organs and tissues to respond quickly to environmental change or internal need. Gene expression here is viewed as a dynamic process, which is continuous and responsive to interactions between biochemical actors in promoting or repressing the activity of genes. Complex interactions between cell and environment result in dynamic feedback loops, creating millions of possible variants of gene activity. Development of the individual is propelled by a series of interactions in which the inherited potential of the fertilised egg diverges through environmental interactions to become realised in the phenotype of the adult. Each of these layers can be thought of as characterised by interactions within and between numerous 'epigenomes' (Gilbert, 2002).

A crucial point in approaching epigenetics developmentally (as opposed to intergenerationally--we will discuss this later in this paper) is that epigenetic changes are envisaged as occurring continuously. The timeframe of epigenesis therefore lies within the timeframe of the individual life of the organism and can be used to explain the rapid emergence and adaptation of unique physical and psychosocial characteristics. In discussing 'DNA memories of early social life' Hoffmann and Spengler (2012) comment '[...] the old-school concepts that genes are "chiseled in stone" or that they alone determine development have been disproven. The discovery of the epigenome provides an explanation, at the molecular level, for how and why early positive and negative social experiences give rise to a biological memory that can have lifelong impacts.' One of the major advances of epigenetics is the introduction of a novel approach to temporality: not only is time reinserted in the otherwise static genomic research but also as time unfolds in the individual organism it always creates new conditions for further development. Every epigenome develops differently because its time is contextual.

In understanding the accumulation of epigenetic changes, there are several biological mechanisms with which the epigenome can be altered to retain 'memories' after exposure to environmental stimuli. Histone modification describes reconfiguration of the topology of the DNA strand in response to cellular activity. This can be used to suppress or promote gene transcription, creating a unique 'histone code', where biochemical interactions with the environment leave unique memory traces at the molecular level with distinct time courses. Theories of histone modification provide a molecular framework for epigenetic approaches to learning that aim to describe how neurons appear to be collectively capable of encoding our thoughts, actions, and interactions with the environment as biologically imprinted alterations. A second means of 'fixing' epigenetic changes is through DNA methylation, where a methyl group 
'covers' the gene and silences its activity by preventing transcription from occurring. DNA methylation is crucial to the control of gene function, and, as with histone modification, is a reversible event that is responsive to external environmental factors.

The cell-specific pattern of epigenetically modulated gene transcription, mediated by DNA methylation and histone modification, therefore provides a more specific definition of the 'epigenome'. Epigenetic change results in the emergence of corporeal 'memories' reflecting our person-specific environment and life experiences. For example, hormones and neurotransmitters such as serotonin and dopamine, which are continuously responsive to environmental stimuli, regulate various aspects of brain activity and are thought to act as sensitive conduits for epigenetic change. This may mean that exposure to severe or chronic trauma or adversity, particularly during early life, has potential to generate enduring epigenetic changes that irrevocably alter neurodevelopment and subsequent brain physiology and personality.

The challenge for social scientists engaged in epigenetics research is to successfully link these two traditionally disparate fields of enquiry (the biological and the social) to create adequate accounts of lived, everyday complexity as it emerges through the social epigenome, that is myriad miniscule interactions that are at once socioculturally and materially, relationally and biologically situated. Such accounts might act as bridges unifying these divergent sciences and thus have significant practical repercussions for understanding and influencing lived human experience. The purpose of this paper is not to provide a review of scientific advances, but to begin, first, to unravel the emergence of the social epigenome as a conceptual space and, second, to discuss methodological obstacles impairing the diffusion and integration of theories of epigenetics with the social sciences.

\section{The quest for causality and the making of social epigenetics}

Social epigenetics is a heavily evolving field of research, not only because it is relatively new, but because models, metaphors and procedures for incorporating social sciences into epigenetic research (and vice versa) have yet to be defined and agreed. Social epigenetics is a field of technoscience in the making, where processes of standardisation and communication take centre stage in acceptance and consolidation amongst mainstream researchers and everyday life (Bowker \& Star, 1999; Busch, 2011; Papadopoulos, 2011a, 2015). However, truly interdisciplinary integration of social science within this purported 'science of social science' also needs to go beyond traditional anthropological commentary and critical observation.

When social science and epigenetics meet the quest is not to better understand the role of genetics in society but to develop a multi-scale epigenetic explanation of complex organisational and social behaviour, from cell to society and back again. The relevance of epigenetics for social science has been documented meticulously (Landecker \& Panofsky, 2013) and the broader cultural and political implications of epigenetics have been discussed elsewhere (Landecker, 2011; Niewohner, 2011; Papadopoulos, 2011b), although they have thus far received relatively little attention in the social sciences (Meloni, 2014). This paper attempts to identify some of the questions and challenges accompanying interdisciplinary integration of social science and epigenetics.

The main scientific tools used to define and elucidate epigenetic pathways have so far focussed heavily on animal experiments, which are conducted under tightly controlled environmental conditions. Studies in humans are less dominant and typically rely on analysis of 
blood, saliva, or tissue samples from groups of individuals exhibiting a specific phenotype, identified with respect to a criterion such as a medical diagnosis, grouped by exposure to a particular environment, and/or twins or families with shared DNA. To date, the primary goal of social epigenetics has been an attempt to define standard aggregates of environmental conditions, biomolecular pathways, and gene expression, a triptych that can be called an 'ecomorph' (Papadopoulos, 2011b). The majority of published original research articles attempt to causally link environmental stimuli to specific epigenetic changes in gene expression, and ultimately, more complex levels of phenotype (Bagot \& Meaney, 2010, p. 756). Much of this research is predicated upon relatively linear notions of causality, within which both the common elements of superficially different environments and the contingent, synergistic interactions of multiple environmental forces tend to remain largely invisible.

Further studies need to be performed to identify complex meaningful environmental exposures and pathways. Without this information the design of studies that correlate epigenetic markers to social-environmental conditions is methodologically and statistically challenging. That epigenesis is primarily considered to be a biochemical phenomenon also raises important ethical and philosophical questions. For example, the view that our mental and emotional health is governed by modifiable epigenomic neurochemical exchanges has profound implications for designing social and medical interventions. To what extent can environmental factors, or personally held, relationally acquired, meanings be reduced to biochemical events? What, if any, are the conduit pathways that causally enable specific sensations to induce predictable, tangible changes in bio-molecular physiology and gene function? How can we meaningfully bridge the lived experience of the body-in-the-world, changes in brain chemistry, and the cellular environment of the gene? In order to begin approaching these questions we will focus on four dimensions of epigenetics research that constitute the most contentious and, simultaneously, promising points of contact between social science and epigenetics: first, the condensation of the environment to a biochemical event; second, the limits of epigenetic studies in humans; third, the temporality of biochemical experience and the question of reversibility of epigenetic alternations; fourth, the heritability of epigenetic changes and its implications for intergenerational accountability.

\section{Animal studies and the condensation of the environment to a biochemical event}

Animal studies have been the workhorses (or rather the 'work rats') of experimental research in epigenetics, but raise significant conceptual issues for translation of findings to the social sciences. Animal studies commonly feature genetically identical (cloned) rodents, where mechanisms for generating epigenetic changes can be triggered and isolated by systematically exposing mice or rats to various stimuli and observing alterations in gene expression and brain chemistry. Psycho-social interventions involve stress experiments such as parental separation, dietary deprivation, use of 'prenatal restraint stress', forced swimming in cold water, and 'social defeat stress paradigm'. A few studies of higher social functioning have been performed in nonhuman primates, however, sample sizes tend to be small, and the advantages of experimenting on non-human primates in a social sciences context are unclear. Animal experiments are frequently combined with pharmacological treatment using gene expression promoters or inhibitors, and cloned 'knock-out' mice with genetically modified sections of DNA. 
For example, basic research recognises the release of what are commonly described as stress hormones as an important mechanism by which social adversity can impact the epigenome (although it should be noted that in social science some see 'stress' as an intrinsically reductionist concept--e.g. Newton (2007). Whilst these pathways also form part of healthy functioning, it is postulated that malfunctions in expression of 'protective genes' that ameliorate environmental adversity may precipitate vulnerability to social and mental health problems. In studies of rats, licking and grooming by the mother was found to release serotonin in the pup's brain which activates serotonin receptors in the hippocampus. These receptors recruit transcription factors to 'turn on' the protective gene that inhibits stress responses, thereby reducing anxiety in adulthood (Zhang et al., 2010; Zhang, Parent, Weaver, \& Meaney, 2004).

An important aspect of animal studies of epigenetics is how tightly controlled the animals' environments need to be. For example, epigenetic researchers conducting drug trials on rats discovered that they had to standardise the handling and caging regimes of the rodents, since their findings suggested that handling variation was a more potent driver of epigenetic variation than the drugs being tested. In this context, the ecologies of living animals involved in the study do not just represent the background scenery on which experimentation takes place; they have to be taken into serious consideration in order to conduct successful experiments. In social sciences research it would clearly be unfeasible to control the environments of human subjects in this way.

A further barrier to identifying potential targets for epigenetic differentiation in the social sciences lies in the way in which 'the environment' is conceptualised. The core experimental arrangement of existing epigenetics research is located at the genetic and bio-molecular level, focusing on biochemical processes such as DNA methylation and histone modification. Viewed from the perspective of the gene, anything else in the cellular environment beyond the DNA is considered environmental. At this level, how the environmental focus of the research (e.g. nutritional, pollution, care, touch, language, social adversity etc.) can be conceptualised and operationalised can vary immensely. Specific aspects of the environment might be erased while others can be over-emphasized. These varying conceptualisations of the environment are then condensed into the biochemical level as causal factors of epigenetic changes. Environments are in this way reduced to biochemical events when viewed from the perspective of the gene.

This condensation of the environment to a biochemical event raises important questions about the ways in which specific environments for a certain study are defined. Biological scientists frequently appear unconcerned about the way in which environmental contexts are conceptualised or the influence of social factors and disciplinary knowledge structures in their selection of candidate pathways of interest. In designing epigenetic social science research, the procedures whereby the social constituents of epigenetic change are quantified will be crucial for understanding the implications of biochemical alterations of the epigenome. This information is largely absent from existing basic science epigenetics research.

To summarise, animal experiments involve rigorous control of environmental conditions, nutrition, and social interactions, providing a key methodology for defining a biological basis of causal relationships in the timing of epigenetic changes. However, rodent models translate poorly to studies of animals in natural environments or to social science studies in humans, which necessarily involve the accumulation and interaction of multiple social and environmental exposures, and a complex social perspective in defining both exposures and outcomes. There remains a vast conceptual gulf between cortisol and serotonin responses in cloned laboratory 
animals living under tightly controlled conditions and the experiential stresses and existential traumas lived by humans in their natural environments. Researchers have barely begun to consider the complexities involved in designing studies which move between these different levels of explanation.

Thus, it should be noted that it is animal studies that provide the clearest evidence that gene-environment interactions exist, confirming causality of epigenetic changes. The findings of animal experiments have clear implications for the design of studies aimed at elucidating the impact of psychosocial factors on gene expression. At the same time, focussing on animal studies in experimental conditions effectively relegates the capacities for cultural transmission, affective communication and meaning-making to a largely subsidiary role. In the process of the condensation of the environment to a biochemical event a lot can be lost. Moreover, studies in humans are most likely to be of a comparative or epidemiological nature, capable of identifying associations between variables, but unable to establish causation. Without strict control over the genetic make-up and precise conceptualisations of the environment of research subjects, it will be difficult to distinguish general epigenetic diversity, or 'noise', from specific changes due to the psycho-social factors of interest.

\section{The limits of epigenetic studies in humans}

Moving away from animal experiments, studies in humans face important challenges in disentangling the epigenetic pathways of interest from genetic and epigenetic heterogeneity within the general population. Statistically, the main issue is 'multiple testing': each epigenomic marker being tested adding considerably to the number of participants required, which quickly results in uneconomically large sample sizes. Although it is tempting to search the entire epigenome for epigenetic variations, this would require data from at least as many individuals as used in Genome Wide Association Studies (GWAS), typically many thousands of subjects. Recruitment targets of this scale are beyond the reach of most individual research groups requiring multicentre collaboration and national bio-banks to facilitate access to biological samples and a unified approach to the collation of demographic and qualitative data. Although sample sizes can be reduced by searching for epigenetic changes at specific sites along the DNA strand, unless strong candidate loci have been identified (e.g. through animal studies) these studies are biased toward pre-existing assumptions about suspected pathways, and will be confined to testing 'the usual suspects' rather than discovering anything new. A clear advantage of storing cell samples within bio-banks would be that samples remain accessible, and can be analysed in future research as knowledge evolves and more relevant candidate loci and pathways are identified. However, it should be noted that bio-banks raise their own distinct ethical and societal challenges (Rose \& Rose, 2012).

One method of limiting genetic and epigenetic variations between human subjects has traditionally involved performing studies with identical twins or families. For example, studies in monozygotic twins, who share identical DNA, are being used to explore epigenetic differences between siblings where the environment is assumed equal (or changing in very specific ways). However, critics have consistently taken issue with the assumption of equal environment highlighting numerous conceptual and evidential concerns (e.g. Joseph, 2003). The equal environment assumption incorporates relatively gross social indicators such as socio economic status and gender but precludes small scale and micro-social variation in the meanings and 
material circumstances of being a twin or non-twin. It has long been challenged by actual studies of monozygotic twin pairs by comparison to both dizygotic pairs and to non-twins (Kringlen, 1967), although advocates of the method instead tend to emphasise heritability indices that estimate degrees of shared environment mathematically rather than assessing it empirically. The relevance of these concerns is highlighted with respect to recent high-profile developments in epigenetics including the Peri/postnatal Epigenetics Twin Study (Loke et al., 2013) and the popularising work of eminent researchers such as Spector (2012).

A significant practical barrier to the study of epigenetics in a social context lies in the inaccessibility of brain tissue samples. Brain tissue is likely to be most relevant to our social relationships, thought processes, memory, and sensory experiences. Ideally it would be possible to obtain brain biopsy samples for examination of cerebral gene expression, but these samples can only be obtained retrospectively via autopsy. Although a few studies involving human brain tissue have been reported in the literature, e.g. comparing the brains of suicide completers to people who died from other causes, studies are rare and tend to be limited to a small number of subjects. Individuals have also been selected retrospectively based on outcome, which is more likely to lead researchers to erroneously identify statistically significant associations. Even where significant epigenetic changes in the brains of individuals are observed it becomes impossible to separate cause from correlation; epigenetic markings could merely correlate to symptom progression, the consequences of medication, or the accumulated effects of related lifestyle changes. Samples of more accessible body fluids, such as blood, are more commonly used in epigenetic studies of living subjects; however, whether epigenetic changes within the blood closely reflect those in brain tissue remains to be established. The closest that we can get to noninvasively obtaining brain tissue samples from the living involves harvesting a swab of cells from the inside of the nose. By sampling from the nose it may become possible to examine neuronal cells, which are more likely to include epigenetic markers that have been influenced by sensory responses and psychosocial factors.

What is common to all epigenetic studies with humans though is the absence of individual experiences as one of the key environmental factors. The environment is usually considered in many epigenetic studies as something objective and given. But a valuable extension of current epigenetic research could include a more complex and nuanced consideration of the environment and how it is experienced by research subjects. Instead of considering only abstract descriptions of the environment the inclusion of specific and discrete experiential realities of people could significantly improve the validity of epigenetic research.

\section{Epigenetic temporalities}

One of the biggest promises of epigenetics research is the insertion of time into biogenetics. Ontogenetic time is crucial for epigenesis, not only because epigenesis is individual specific but because time is productive in the sense that it always creates new and different conditions that then become the starting point for the next phase of development. As epigenetic instructions for development of an embryo begin to unfold, the in utero environment has potential to influence further stages of fetal development. 'Fetal and neonatal life are characterized by tremendous plasticity and the ability to respond to environmental factors (nutrients, oxygen, hormones) by altering gene expression levels via epigenetic modifications' (Heerwagen, Miller, Barbour, \& Friedman, 2010). First and second trimester environmental factors are thought to include 
infections such as rubella, influenza, and toxoplasmosis, as well as social factors associated with maternal environmental adversity. Other proposed prenatal factors include protein and nutritional deficiencies, inflammation, obstetrics complications and fetal distress. Early life and adolescence are particularly susceptible to environmental and social influences, and since moments of epigenetic transformation may be fleeting, their putative effects are often presumed to remain quiescent, becoming magnified only later in adulthood.

Brain architecture is thought to develop during 'sensitive periods', 'each of which is associated with the formation of specific circuits that underlie specific abilities' (Hoffmann \& Spengler, 2012). Biological pathways regularly stimulated by acute psychosocial stress, or chronic exposure to toxins or recreational drugs, are speculated to become epigenetically 'hard-wired' by embedding permanent changes in DNA methylation. However, the timescale for these changes to occur is currently unclear. In any case, researchers seem to agree that a synergy between genes and environment occurs, and depending on the timing of epigenetic changes during development their effects may be latent, reversible or permanent. As we age, the epigenome is thought to tend toward a state of dynamic equilibrium, emphasizing routine day to day functions in response to internal signals. It is proposed that studies of epigenesis may help to untangle complex interactions as they unfold over time (Gilbert \& Epel, 2009). However, as this field is still very young, epigenetics researchers have not so far had an opportunity to examine and standardise longitudinal changes across the lifetime of individuals.

The design of studies to examine the 'biochemicalisation' of individual experience become more complex when we consider that the timing of exposure to environmental triggers appears to be a crucial factor in determining the impact of epigenetic interactions. In social science research, a benefit of implementing a longitudinal study design, where epigenetic testing is performed at multiple time-points, is that individuals act as their own controls, which vastly reduces the number of subjects required. Also, in examining models based on genetic profiling and personalised medicine, researchers move away from a reliance on cohort studies for the evaluation of average behaviour to a much more tailored approach. Although time, as such, is not a central consideration in most basic science studies, this is likely to become a major feature of social epigenetics research. Ideally, studies would follow subjects from birth, or incorporate prospective recruitment of subjects approaching key moments of transition. If subjects can be identified prospectively, prior to exposure, epigenetic changes associated with particular 'social pathogens', or accumulated over an entire lifetime, could be explored. Although randomisation to particular drugs, therapies, or other interventions, has been widely used to establish causality within evidence-based medicine, it is unlikely that a randomised trial design would be appropriate to the majority of social sciences studies. The vast majority of research appears likely to remain observational.

In social epidemiology, environmental factors considered for epigenetic associations include 'urbanicity', adverse migration experiences and low socio-economic status (mimicked by 'social defeat' or 'unstable social hierarchy stress' in animal studies). However, it can be difficult to see how to convincingly link epidemiological findings and social risk factors, such as for example negative migrant experiences upon arrival or urban vs rural environment, to epigenetic changes (Brown, 2011; Spauwen \& Van Os, 2006). So far, the evidence for a link between life changing events (and the experiencing of these events by the individual) to changes in gene expression is largely circumstantial. Moreover, the practices of methodological individualism presumed within many biologically-driven studies necessarily preclude from rigorous 
consideration those emergent properties of naturally-occurring (rather than experimenter constituted) groups and their everyday activities. Whilst these properties and activities are often seen within social science as significantly mediating and constituting lived experience, they are difficult to be included within the experimental designs typically preferred in epigenetic studies.

One area where randomised trials are likely to predominate for translation of epigenetics research is in the field of pharmacogenetics. Reversibility of epigenetic processes is already a dominant question in basic science research, and the development of personalised epigenetic drugs is likely to become a key driver for clinical translation. Studies have shown that behavioural epigenetic changes in mature rats could be reversed by injection of drugs into the brain. These studies tend to view epigenetic processes from a normative perspective aiming to identify epigenome-biochemical-phenotype pathways that can be altered to ameliorate or counter widespread pathologies or to erase and recalibrate traces of bad epigenetic modifications.

Epigenetic alteration of gene expression can either be protective or harmful depending on context (ie phenotypic traits that are later deemed socially dysfunctional might once have been adaptive). However, the idea of drawing a clear distinction between 'good' (healthy) and 'bad' (pathological) interactions between the gene and the cellular environment is challenging. Reversibility of epigenetic interactions, either through pharmacotherapy or through targeted alteration of environmental conditions that have negative effects on the individual, is the closest that epigenetics has so far come to becoming an applied science. But despite its potentials this approach is not unproblematic. The last few years of research into the pharmacogenomics of psychiatric disorders, for example, have seen the first attempts at genetic personalisation of drugs categorised as antipsychotic treatment, including prediction of side-effects (Cacabelos \& Martinez-Bouza, 2011; Daray, Maffia, Rothlin, \& Errasti, 2012; Kirchheiner, Seeringer, \& Viviani, 2010; Zandi \& Judy, 2010). Since successful responses to pharmacological strategies are likely to be in part epigenome dependent, personalised genetic and epigenetic mapping are thought to be required for adverse epigenetic effects to be successfully treated. To the extent that theories of epigenetics promise to deliver a better understanding of the making of the individual within society, pharmacogenomics is expected to offer insights into its 'unmaking'. Although positive epigenetic modifications may well be driven by social or micropolitical forces, current research mainly focuses on responding to negative epigenetic change, rather than upon prevention. The search for a 'cure' to the ills of society appears to be almost overwhelmingly assumed to be pharmaceutical, rather than, for example, relational, social or psychotherapeutic.

But the issue of reversibility and its translational potential is also from an epistemic perspective controversial. The idea that 'negative' epigenetic changes may be chemically reversible in adulthood (Szyf, McGowan, \& Meaney, 2008) seems to be in contradiction with the prevalent understanding of time in basic epigenetic research: epigenesis considers time as essentially irreversible. In ecological-biological biology (Gilbert \& Epel, 2009) time is irreversible because the epigenetic changes that occur in each specific moment constitute a unique starting ground for subsequent changes. Since these changes depend on the concrete interactions between the environment and the organism they are always different. Time creates always new and specific developmental conditions. Epigenesis in ontogeny is contingent and probabilistic (Gottlieb, 1992). The hope that through translational research epigenetic modifications can be erased or recalibrated is based on the implicit assumption that the embryonic genome exists in some ideal state of 'purity', which later becomes epigenetically corrupted or dysfunctional. That genes activated or deactivated by physiological responses to social or environmental adversity 
might be restored to their ancestral state through pharmacotherapy defies the idea that it is not only the creativity of the environment but also the creativity of developmental time that drives epigenesis.

\section{Heritability of epigenetic changes}

On a longer timescale, the focus of epigenetics shifts both temporally and physically to the key question of whether epigenetic markings may be transmitted to offspring. Epigenetic modifications to a parent's DNA have been suggested to be passed to their children via 'genetic imprinting'. For example, Yehuda and Bierer (2008) found lower cortisol levels and a higher incidence of diagnoses of post-traumatic stress syndrome and anxiety disorder in the adult offspring of Holocaust survivors than in a genetically similar control group and hypothesize that this could be epigenetically imprinted, rather than the effect of a 'shared environment'. Similar findings have been observed in the descendants of first generation migrants. This, and other research, has led to speculation that genetic social memories of potential past traumatic events may leave traces of neurochemical responses that permeate the physiology and mental health of future generations, although, of course, alternative explanations remain plausible (many Holocaust survivors and migrants will bequeath multiple environmental and social expectations, resources and challenges to their offspring, whatever epigenetic modifications they might also transmit). From this perspective, an embryo can be considered as having been epigenetically influenced by the environment of its parents (and grandparents) from the first moment of conception.

Implicitly, there are issues of intergenerational responsibility and ethical accountability which this research raises. For example, nutritional epigenetics shows how research associating pregnant women's dietary deficiencies in folic acid to neural tube defects (e.g. spina bifida and anencephaly) is permeated by various presuppositions about food and health that ultimately converge upon 'maternal metabolism as the intersection of food, food regulation, nutrition as medicine, self-regulation, ideas of intervention, hormonal regulation and the heritability of patterns of gene regulation' (Landecker, 2011, p. 184). Similarly, Juengst, Fishman, McGowan, and Settersten Jr (2014) note the increasing prevalence of health risk messages that extrapolate from findings in rodent population to suggest that pregnant women may be responsible for their future child's obesity. Pregnant women thus become the effective target of a set of intersecting and ideologically-inflected societal concerns that get recast, for them, during their pregnancy, as a largely personal responsibility that is biochemically transmitted through the epigenome toward future generations. In the case of folic acid deficiencies whose consequences are well established and often severe, but easily avoided using a precisely targeted intervention (dietary supplements) this is arguably permissible. In relation to obesity, by contrast, the scientific evidence is far less clear, whilst the simultaneous concordance of this advice with the neoliberal responsibilisation that Cederstrom and Spicer (2015) describe as the 'wellness syndrome' is transparent.

Similarly, issues pertain to the intergenerational risk of diagnoses of anxiety disorder and PTSD, where causality is uncertain and diffuse, multiple biological pathways have been implicated, and there are no simple interventions--whether dietary, pharmaceutical, behavioural, psychotherapeutic or societal--that have been consistently demonstrated to either prevent or remedy the experiences associated with these diagnoses. The inferred responsibilities of pregnant women from traumatised generations or of migrant status, for example, are here far more 
continuous and diffuse, whilst the likely consequences of any mitigating steps they might take are far less certain. The intertwined practical and the ideological implications of epigenetic research consequently loom much larger in relation to these diagnoses not only because there are no single environmental factors that have caused these epigenetic changes but also because people simply cannot know where their accountability sensibly ends within these complex conditions.

\section{Conclusion: disciplinary integration and managing the flows of epigenetic knowledge}

Social epigenetics has potential to transform the social sciences. However, research has so far been limited due to barriers between distinct groups of specialists, each contributing their own tools, insights, language and methodology to our understanding of 'epigenetic' processes. Within each layer of the epigenetic puzzle, different groups of researchers adopt differing visualisations, methodologies and language to discuss and interpret their research. Ideas surrounding epigenetics, formulated at the biomolecular level, are beginning to permeate into other disciplines. However, in order to develop a constructive dialogue between basic scientists and social scientists an increased exchange of concepts and tools between disciplines (as well as different approaches inside these disciplines) would be necessary. At present, the absence of consistently established genetic loci and biomarkers for environmental interactions makes it difficult to see a clear pathway for integration of epigenetic markers and social science research.

Epigenetic theories seem to complement what has already been gleaned from some psychiatric, psychological, sociological and epidemiological studies, that pathways are intensely individual, temporal, varied, contingent and multi-factorial. Thus, if one of the aims of current epigenetics is its translational capacity, the question here is which disciplines and imperatives involved in epigenetic research are driving translation, and in what direction. This question perhaps acquires particular force at a time of 'austerity', when public funding for research is restricted and policy frequently emphasises scientific, technological and mathematical disciplines as its most suitable recipients. The common view of the translational process as following an inflexible path from gene mapping - gene expression - biochemical pathways - environmental triggers - pharmacology - society offers a simplistic and limited understanding of the interconnections between different disciplines.

Both our reading of the literature and our analysis of its methods suggest that crossdisciplinary integration and potential for translational research are not connected in a linear way. Rather, like parallel epigenomes, each of these disciplines changes, and is connected, through multiple relations to the others. In relation to sociology and other social sciences, epigenetics simultaneously appears as both an opportunity and a threat. In promising to repudiate genetic determinism and reductionism, and open up the biosciences to collaborations that wholly incorporate the nuance and complexity of social structures and relations and their consequences for health, wellbeing, productivity and life satisfaction, epigenetics appears as a distinct opportunity: moreover, one arising at a moment in the political economy of the academy when many social scientists feel that they must strive as never before to establish the continued relevance and impact of their work.

Simultaneously, epigenetics contains distinct potentials to reconfigure the effects of the social realm as effectively reducible to diverse sets of molecular changes that--unlike say the shifting, context dependent meanings of being unemployed or homeless, of migrating to escape persecution or of experiencing racism--are apparently definable and measurable and without any 
attendant presumptions of value. Epigenetics therefore contains potentials for reductionism, notwithstanding that the forms of reductionism it facilitates may well be new, Lamarckianinflected ones, epistemically distinct from those that accompanied the genetic thinking of the last century (Meloni \& Testa, 2014). Epigenetics and its current applications contain many risks and promises, so the open question that therefore arises is not primarily to critique epigenetic knowledge or investigate how social epigenetics will be generated but to actively engage in research on social epigenetics and in the management of the flows of epigenetic knowledge.

At the same time, we wish to emphasise again that when the devils of determinism and reductionism persist it is frequently because they are lurking in the fine details of practice. The field of epigenetics might bring significant innovation and radical improvements towards previous genocentric approaches but it all depends in the concrete and specific ways research is designed and conducted. The relevant details, here, are those involved in pursuing appropriately detailed investigations of meaningful human experience and organic epigenetic process, side by side and on equal terms. We have seen that there are obstacles to the epigenetic strand of such investigations because they cannot measure degrees of epigenetic marking in the living brain and must instead rely on proxy measures. However, our primary concern here is with the details associated with experience and the measurement of meaningful human activity -- such as would be required, for example in the conduct of any valid social or cultural epigenetics (for example, Jablonka this volume).

We should first note that the degree of complexity involved in such an endeavour is sometimes obviated by the superficially neutral equation of 'meaningful activity' with 'behaviour'. Briefly, meaningful activity is experiential and often reflexive. It involves interpretive orientation toward (often implicit) shared cultural norms, values and standards; behaviour, by contrast, is what we simply find ourselves - or, more accurately, our bodies - doing for us. Treating behaviour as simply equivalent to meaningful activity seemingly eliminates the need to grapple in anything other than relatively superficial ways with questions of what human experience is, of what it consists, and of how for humans it always includes vital relational, material and cultural constituents (Cromby, 2015a; Stephenson \& Papadopoulos, 2006). Hence this equation is already either a reduction (that strips away some meaning) or a covert re-specification (that imposes researcher-defined meanings), one which in either case tends to render rationally invisible at least some of the continuous dynamic influences of history, biography, place, situation, context, relationship intention and purpose, and so to occlude the ways in which human experience is always the outcome of these culturally-imbued influences just as much as it is the result of organismic processes.

Moving to consider the fine details of research practice we do not of course leave these conceptual matters behind: rather, we see them codified and reproduced as deep assumptions within methods and procedures. The continuous need for quantification of human experience and meaningful activity (to render it as a variable that can then be equally compared to, say, levels of methylation) impels a series of decisions with ongoing ramifications for the status of findings. Two examples will briefly illustrate this. First, in this article we have already seen how some studies rely on functional psychiatric diagnostic categories such as PTSD and anxiety disorder. Such studies frequently (but not always) naturalise these categories, treating them as actual diseases or conditions that can be diagnosed with acceptable levels of reliability and validity. Yet the problems attendant upon functional psychiatric diagnosis are so widely recognised that in the USA the National Institute of Mental Health (who distribute relevant 
research funding) announced in 2013 that their future funding strategy would no longer depend upon it, and that they would instead strive to develop their own more robust taxonomy. Second, psychometric measures such as questionnaires and associated rating scales are often used to convert fluid, situated psychological qualities and experiences into quantifiable variables. But there is evidence that these instruments, themselves cultural artefacts, are in fact actively and differentially interpreted by study participants, to the extent that "it is a misplaced assumption that participants have direct access to their response and that this response is static and can be represented as a mark along a line ... rating scale data, despite being statistically manipulated, should not (and indeed cannot) be thought of as objective" (Rosenbaum \& Valsiner, 2011, p. 61).

Vitally, we do not raise these concerns because we wish to simply challenge epigenetic research. Possibly the reverse is the case: we want to challenge reductionist methodologies in the social sciences that can be used to inform epigenetic research. In other words, we wish to strengthen the potentials of epigenetics by using specific traditions in the social sciences that could provide solutions to adequately approach human experience in non-reductionist ways. Certain traditions in social science have established knowledge and expertise in the ways that society, history and culture can be identified, assessed and compared with other sources of evidence. We are looking for a meeting point between social epigenetics and social science where the social and cultural aspects of human life are not reified or reduced to single monodimensional indices; correspondingly it will be more likely to recognise that -- as process (Kleinman, 2004) -- society and culture are likely to demand forms of assessment and analysis that are both theoretically sophisticated and empirically sensitive.

Social epigenetics need a social science that works with the fine detail of the dynamic play of meaning and experience, rather than abstracting and codifying them and thus rendering them relatively static as variables. We do not attempt here to substitute quantitative research by qualitative studies or revive persistent dichotomies; rather, we highlight the centrality of human experience and its significance not only in generating new hypotheses but also in potentially accounting for unexplained variance (by mapping the contingent, dynamic vagaries of lived experience) and thus contributing to improved future studies. We are thinking here of mixed and innovative methodologies and interdisciplinary research designs. Many traditions in the social sciences have considered in depth the workings, practices, paradoxes and effects of the thoroughgoing transdisciplinarity that successful epigenetic research demands (e.g. Cromby, 2015b), including formulating guidelines for its more effective conduct. So there is, to conclude, a distinct and much-needed role for social science in social epigenetics. But social science will have to confront itself and change in order to be able to be up to this task. Equally, social epigenetics will have to change as they incorporate methods and insights on human experience provided by social science. Whether many social scientists and epigeneticists will take up these challenges, and whether this transdisciplinary synergy can contribute to non-reductionist understandings of human life and experience is yet to be decided. We are still a long way from a 'science of social science'.

\section{$\underline{\text { References }}$}


Bagot, R. C., \& Meaney, M. J. (2010). Epigenetics and the biological basis of gene $\mathrm{x}$ environment interactions. J Am Acad Child Adolesc Psycbiatry, 49(8), 752-771. doi:10.1016/j.jaac.2010.06.001

Bowker, Geoffrey C., \& Star, Susan Leigh. (1999). Sorting things out: classification and its consequences. Cambridge, Mass.: MIT Press.

Brown, A. S. (2011). The environment and susceptibility to schizophrenia. Prog Neurobiol, 93(1), 23-58. doi:10.1016/j.pneurobio.2010.09.003

Busch, Lawrence. (2011). Standards: recipes for reality. Cambridge, MA: MIT Press.

Cacabelos, R., \& Martinez-Bouza, R. (2011). Genomics and pharmacogenomics of schizophrenia. CNS Neurosci Ther, 17(5), 541-565. doi:10.1111/j.1755-5949.2010.00187.x

Cederstrom, C., \& Spicer, A. (2015). The Wellness Syndrome. Cambridge: Polity Press.

Cromby, John. (2015a). Feeling Bodies: Embodying Psychology. London: Palgrave Macmillan.

Cromby, John. (2015b). Interdisciplinarity: Reconciling the irreconcilable? Canadian Medical Association Journal, 187(13), 998-999. doi:10.1503/cmaj.150372

Daray, F. M., Maffia, P. C., Rothlin, R. P., \& Errasti, A. E. (2012). Pharmacogenetics in psychiatry: How far are we from clinical application?]. Vertex, 23(104), 299-309. Retrieved from http://www.ncbi.nlm.nih.gov/pubmed/23170302

Gilbert, Scott F. (2002). The Genome in Its Ecological Context. Philosophical Perspectives on Interspecies Epigenesis. Annals- New York. Academy of Sciences, 981, 202-218.

Gilbert, Scott F., \& Epel, David. (2009). Ecological developmental biology : integrating epigenetics, medicine, and evolution. Sunderland, Mass.: Sinauer Associates.

Gottlieb, Gilbert. (1992). Individual development and evolution : the genesis of novel behavior. New York: Oxford University Press.

Heerwagen, M. J., Miller, M. R., Barbour, L. A., \& Friedman, J. E. (2010). Maternal obesity and fetal metabolic programming: a fertile epigenetic soil. Am J Physiol Regul Integr Comp Physiol, 299(3), R711722. doi:10.1152/ajpregu.00310.2010

Hoffmann, A., \& Spengler, D. (2012). DNA memories of early social life. Neuroscience. doi:10.1016/j.neuroscience.2012.04.003

Jablonka, Eva, \& Lamb, Marion J. (2005). Evolution in four dimensions. Genetic, epigenetic, behavioral, and symbolic variation in the history of life. Cambridge, Mass.: MIT Press.

Joseph, J. (2003). The Gene Illusion: genetic research in psychiatry and psychology under the microscope. Ross on Wye: PCCS Books.

Juengst, Eric T., Fishman, Jennifer R., McGowan, Michelle L., \& Settersten Jr, Richard A. (2014). Serving epigenetics before its time. Trends in Genetics, 30(10), 427-429. doi:http://dx.doi.org/10.1016/j.tig.2014.08.001

Kirchheiner, J., Seeringer, A., \& Viviani, R. (2010). Pharmacogenetics in psychiatry--a useful clinical tool or wishful thinking for the future? Curr Pharm Des, 16(2), 136-144. Retrieved from http://www.ncbi.nlm.nih.gov/pubmed/20205659

Kleinman, A. (2004). Culture and Depression. New England Journal of Medicine, 351(10), 951-953.

Kringlen, E. (1967). Heredity and environment in the functional psychoses: case histories. Oslo: Universitetsforlaget.

Landecker, H. (2011). Food as exposure: Nutritional epigenetics and the new metabolism. BioSocieties, 6(2), 167-194.

Landecker, H, \& Panofsky, Aaron. (2013). From Social Structure to Gene Regulation, and Back: A Critical Introduction to Environmental Epigenetics for Sociology. Annual Review of Sociology, 39(1), 333-357. doi:10.1146/annurev-soc-071312-145707

Loke, Yuk Jing, Novakovic, Boris, Ollikainen, Miina, Wallace, Euan M., Umstad, Mark P., Permezel, Michael, . . . Craig, Jeffrey M. (2013). The Peri/Postnatal Epigenetic Twins Study (PETS). Twin Research and Human Genetics, 16(Special Issue 01), 13-20. doi:doi:10.1017/thg.2012.114

Meloni, Maurizio. (2014). Biology without Biologism: Social Theory in a Postgenomic Age. Sociology, 48(4), 731-746. doi:10.1177/0038038513501944

Newton, T. (2007). Nature and Sociology. London: Routledge.

Niewohner, J. (2011). Epigenetics : Embedded bodies and the molecularisation of biography and milieu. BioSocieties, 6, NUMB 3, 279-298.

Papadopoulos, Dimitris. (2011a). Alter-ontologies: Towards a constituent politics in technoscience. Social Studies of Science, 41(2), 177-201. doi:10.1177/0306312710385853

Papadopoulos, Dimitris. (2011b). The Imaginary of Plasticity: Neural Embodiment, Epigenetics and Ecomorphs. Sociological Review, 59(3), 432-456. doi:10.1111/j.1467-954X.2011.02025.x 
Papadopoulos, Dimitris. (2015). From Publics to Practitioners: Invention Power and Open Technoscience. Science as Culture, 24(1), 108-121. doi:10.1080/09505431.2014.986322

Rose, H., \& Rose, S. (2012). Genes, Cells and Brains: the promethean promises of the new biology. London: Verso.

Rosenbaum, P., \& Valsiner, J. (2011). The un-making of a method: From rating scales to the study of psychological processes. Theory and Psychology, 21 (1), 47-65.

Spauwen, J., \& Van Os, J. (2006). The psychosis proneness: psychosis persistence model as an explanation for the association between urbanicity and psychosis. Epidemiol Psicbiatr Soc, 15(4), 252-257. Retrieved from http://www.ncbi.nlm.nih.gov/pubmed/17203616

Spector, T. (2012). Identically Different: Why You Can Change Your Genes. London: Orion Publishing.

Stephenson, Niamh, \& Papadopoulos, Dimitris. (2006). Analysing everyday experience. Social research and political change. London: Palgrave Macmillan.

Szyf, M., McGowan, P., \& Meaney, M. J. (2008). The social environment and the epigenome. Environ Mol Mutagen, 49(1), 46-60. doi:10.1002/em.20357

Waddington, C. H. (2012). The epigenotype. 1942. Int J Epidemiol, 41(1), 10-13. doi:10.1093/ije/dyr184

Yehuda, R., \& Bierer, L. M. (2008). Transgenerational transmission of cortisol and PTSD risk. Prog Brain Res, 167, 121-135. doi:10.1016/S0079-6123(07)67009-5

Zandi, P. P., \& Judy, J. T. (2010). The promise and reality of pharmacogenetics in psychiatry. Psychiatr Clin North Am, 33(1), 181-224. doi:10.1016/j.psc.2009.12.001

Zhang, T. Y., Hellstrom, I. C., Bagot, R. C., Wen, X., Diorio, J., \& Meaney, M. J. (2010). Maternal care and DNA methylation of a glutamic acid decarboxylase 1 promoter in rat hippocampus. J Neurosci, 30(39), 13130-13137. doi:10.1523/JNEUROSCI.1039-10.2010

Zhang, T. Y., Parent, C., Weaver, I., \& Meaney, M. J. (2004). Maternal programming of individual differences in defensive responses in the rat. Ann N Y Acad Sci, 1032, 85-103. doi:10.1196/annals.1314.007 